\title{
Incidence of Primary Biliary Cholangitis in a Rural Midwestern Population
}

\author{
Rajan Kanth, MD; Ram Babu Shrestha, MS; Indira Rai, MD; \\ Jeffrey J. VanWormer, PhD; and Praveen K. Roy, MD
}

Background: Primary biliary cholangitis (PBC) is a rare disease with incidence that varies with time and geography. Only two studies have assessed PBC incidence in the United States, with the most recent appearing over a decade ago. The objective of the present study was to assess PBC incidence in the United States in a more recent era.

Methods: The incidence of PBC was assessed in a population-based cohort in rural, Midwestern Wisconsin over two decades spanning from June 1992 through June 201I. Cases were initially identified in the electronic medical record and then manually verified for inclusion according to the American Association for the Study of Liver Disease criteria for PBC.Additional data were abstracted for verified cases.

Results: A total of 79 cases of PBC were identified over the 20-year period for an overall age- and sex-standardized incidence of 4.9 cases per 100,000 person-years. Incidence was higher in females, but changes over time were not significant. After a mean 7.3 years follow-up, all-cause mortality of those with PBC was $29 \%$, and estimated 10 -year survival was $76 \%$.

Conclusions: The overall incidence of PBC in a Midwestern population of the United States has remained relatively stable over the last two decades. Patients have better prognosis, and the survival of PBC cases has improved.

Keywords: Primary Biliary Cholangitis; Primary Biliary Cirrhosis; Incidence; Population-Based Study

$\mathrm{P}$ rimary biliary cholangitis (PBC) is a chronic, progressive cholestatic autoimmune disease characterized by destruction of small intrahepatic bile ducts. It is believed that PBC affects 10-times as many women as men, with a median age at diagnosis of 50 years. ${ }^{1-3}$ The disease progresses over decades and may eventually lead to cirrhosis and liver failure. ${ }^{4-6} \mathrm{PBC}$ was formerly known as primary biliary cirrhosis, but the name was changed from 'cirrhosis' to 'cholangitis' to remove the stigma associated with cirrhosis, as many patients are free of cirrhosis at the time of diagnosis. ${ }^{7}$

The etiology of PBC is thought to be a function of both genetic and environmental factors. ${ }^{89}$ A relatively rare disease, knowledge of PBC has evolved slowly over recent decades.

Corresponding Author: Rajan Kanth, MD, Department of Gastroenterology, Carilion Clinic, 3 Riverside Circle, Fourth Floor, Roanoke,VA 24016. Tel: 540-224-5170, Fax: 540-985-9418, E-mail: rkanth@carilionclinic.org.
Epidemiologic studies suggest that the prevalence of $\mathrm{PBC}$ is subject to considerable geographic variation. Populationbased studies of PBC prevalence have been conducted in the United Kingdom, Finland, Sweden, the United States, Japan, and China, with prevalence rates ranging from as low as 27 cases per million people in Japan to as high as 492 cases per million people in China. ${ }^{1,10-16}$ In the United States, data from Olmsted County, Minnesota from 1975 to 1995 estimated the prevalence of $\mathrm{PBC}$ at 400 cases per million people. ${ }^{12}$ Data from 1984 to 2000 among Alaska Natives estimated the prevalence of $\mathrm{PBC}$ at 160 cases per million people. ${ }^{17}$

Some evidence suggests that $\mathrm{PBC}$ prevalence may be increasing over time, as rates in northern England rose from 201 cases per million people in 1987 to 336 cases per million

\footnotetext{
Received: November 22, 2017

Revised:April 3, 2017

Accepted:April 24, 2017

doi: $10.3121 / \mathrm{cmr} .2017 .1351$
}

Grant Support: Supported by grant ULITR000427 from the Clinical and Translational Science Award (CTSA) program of the National Center for Advancing Translational Sciences, NIH. Additional research support was provided by the Marshfield Clinic Research Foundation and its generous donors. 
people in 1994. ${ }^{11}$ The previous U.S. studies from over a decade ago by Kim et $\mathrm{al}^{12}$ and Hurlburt et $\mathrm{al}^{17}$ did not indicate increasing $\mathrm{PBC}$ rates, though this was not possible to statistically examine in the relatively small Alaskan population. The purpose of the present study was to provide an update on the epidemiology of $\mathrm{PBC}$, including incidence and prevalence estimates, over a 20-year timeframe in a rural Midwestern population.

\section{Methods}

Setting

The Marshfield Epidemiologic Study Area (MESA) is a geographically-defined region of 24 ZIP codes in central and northern Wisconsin. It was established in 1991 as a dynamic, population-based cohort for epidemiologic research. ${ }^{18}$ Nearly all residents get their health care at the Marshfield Clinic main campus, one of its satellite clinics, or affiliated hospitals. The clinical records of MESA cohort members are available through an extensive electronic medical record system and data warehouse archive. Validation surveys indicate that Marshfield Clinic data systems capture over $95 \%$ of residents' inpatient and outpatient visits, including most all deaths, within the central 14 ZIP codes surrounding Marshfield, with coverage slightly lower among the remaining 10 ZIP codes in the north. ${ }^{19}$ Automated daily review of Marshfield Clinic computerized databases to track cohort membership is supplemented by utility records and public-use data sources, including state and national death files, to help identify people who have left the cohort. This study was approved by the Institutional Review Board.

\section{Case Identification and Validation}

All individuals, 18 years and older, diagnosed with PBC between June 1992 and June 2011 were retrospectively identified. Data were obtained from the MESA database using a broad ascertainment strategy involving International Classification of Disease, version 9 (ICD-9) system code data. The following ICD-9 codes were used: 571.6 (biliary cirrhosis), 571.5 (cirrhosis of liver without mention of alcohol), 571.8 (other chronic nonalcoholic liver disease), and 571.9 (unspecified chronic liver disease without mention of alcohol). Once the final set of potential cases was established, each record was manually reviewed and validated for the diagnosis of PBC.

Table 1. Laboratory Results ${ }^{a}$

\begin{tabular}{|c|c|c|}
\hline & $\mathbf{N}^{\mathbf{b}}$ & Mean (Range) \\
\hline Age (years) & 79 & $58(27-93)$ \\
\hline BMI $\left(\mathrm{kg} / \mathrm{m}^{2}\right)$ & 73 & $30(18-53)$ \\
\hline Follow-up visits & 79 & $88(6-252)$ \\
\hline Albumin (g/dl) & 79 & $4(2-5)$ \\
\hline ALP $(U / L)$ at diagnosis & 79 & $350(57-1305)$ \\
\hline ALP $(U / L)$ at 1 year follow-up & 76 & $271(50-1083)$ \\
\hline ALT (U/L) & 75 & $75(6-267)$ \\
\hline AST (U/L) & 76 & $66(19-245)$ \\
\hline Bilirubin (mg/dl) & 79 & $1(0.2-6)$ \\
\hline Cholesterol (mg/dl) & 53 & $219(93-404)$ \\
\hline Creatinine (mg/dl) & 72 & $1(0.5-1)$ \\
\hline Gamma-glutamyl transpeptidase (U/L) & 40 & $321(16-1071)$ \\
\hline HDL (mg/dl) & 50 & $63(18-155)$ \\
\hline $\lg A(m g / d l)$ & 79 & $206(7-782)$ \\
\hline $\lg G(\mathrm{mg} / \mathrm{dl})$ & 39 & $1624(527-3270)$ \\
\hline $\lg M \quad(m g / d l)$ & 38 & $418(111-1060)$ \\
\hline INR & 54 & $1.2(0.8-3.4)$ \\
\hline LDL (mg/dl) & 49 & $137(32-316)$ \\
\hline $\mathrm{Na}(\mathrm{mmol} / \mathrm{L})$ & 70 & $138(129-143)$ \\
\hline Prothrombin time (sec) & 30 & $15(9-34)$ \\
\hline Triglycerides (mg/dl) & 50 & $121(40-329)$ \\
\hline \multicolumn{3}{|c|}{$\begin{array}{l}\text { BMI, body mass index; ALP, alkaline phosphatase; ALT, alanine transaminase; AST, aspartate } \\
\text { transaminase; HDL, high density lipoprotein; INR, international normalized ratio; LDL, low densit } \\
\text { lipoprotein; } \mathrm{Na} \text {, sodium. } \\
\text { aAll values are reported as recorded at diagnosis unless otherwise indicated. }\end{array}$} \\
\hline
\end{tabular}


Table 2. Patient, Disease, and Treatment Characteristics

\begin{tabular}{lc}
\hline Characteristic & N (\%) \\
\hline Female gender & $75(95)$ \\
Caucasian race & $78(99)$ \\
Edema & $44(56)$ \\
Esophageal varices, portal hypertension, or ascites & $18(23)$ \\
Encephalopathy & $9(11)$ \\
Sjogren's syndrome & $6(8)$ \\
CREST syndrome & $4(5)$ \\
Rheumatoid arthritis & $7(9)$ \\
Osteopenia and osteoporosis & $33(42)$ \\
Psoriasis & $5(6)$ \\
Ursodeoxycholic acid (UDCA) & $79(100)$ \\
Liver transplant & $4(5)$ \\
Death & $23(29)$
\end{tabular}

CREST Syndrome: calcinosis, Raynaud phenomenon, esophageal dysmotility, sclerodactyly, and telangiectasia

\section{Case Definition}

PBC diagnoses were reviewed and confirmed based on the following American Association for the Study of Liver Diseases (AASLD) ${ }^{8}$ criteria: (A) biochemical evidence of cholestasis with elevation of alkaline phosphatase activity for more than 6 months; (B) presence of anti-mitochondrial antibody (AMA); and (C) histopathological evidence of granulomatous destruction of small to medium-sized interlobular bile ducts when a biopsy was performed. The first author determined $\mathrm{PBC}$ case confirmations.

\section{Chart Abstraction}

Detailed data abstraction was conducted using a standardized form for the 79 individuals who had a confirmed diagnosis of PBC. Information abstracted included demographics (age, date of birth, gender, and race); laboratory values (liver enzymes, renal function tests); medication use [ursodeoxycholic acid (UDCA, ursodiol)]; medical comorbidities (hepatitis B, hepatitis C, systemic lupus erythematosus, rheumatoid arthritis); complications of cirrhosis (portal hypertension, variceal bleed, encephalopathy); and death at final follow-up. When documentation was insufficient, variables were noted as "missing or undocumented."

\section{Statistical Analyses}

Analytical procedures were conducted using SAS Version 9.3 (Cary, NC) and generally paralleled those used by Kim et a ${ }^{12}$ in their prior study of PBC epidemiology in southern Minnesota. PBC incidence rates were stratified by sex and estimated in 5-year time periods, including 1992-1996, 1997-2001, 2002-2006, and 2007-2011. Incidence rates were calculated by dividing the number of newly observed PBC cases by the total person-time in MESA during each 5-year time segment. All MESA person-time contributors were considered at-risk. Sex-specific estimates were then directly age-standardized to the distribution of Caucasians in the year 2000 US Census population. A test for time trend was run using a general linear model and assuming a Poisson distribution. A Kaplan-Meier survival analysis was also conducted on PBC cases to examine time to endpoint, death (event), or last known follow-up (censored), per medical records observations.

\section{Results}

There were 79 incident PBC cases observed in MESA between 1992 and 2011. Mean age at diagnosis was 58 years, and nearly two-thirds of the patients were between 40 and 69 years of age. Only four individuals $(5 \%)$ were male and one (1\%) was non-white. Descriptive and clinical characteristics of all PBC cases are outlined in Tables 1 and 2 . Antimitochondrial antibody (AMA) titers ranged from 1:20 to 1:5120, and antinuclear antibody (ANA) titers ranged from $1: 80$ to $1: 640$. Liver biopsy was done in $39 / 79(50 \%)$ cases, of which $9(11 \%)$ were histological stage $1,12(15 \%)$ were stage 2, $11(14 \%)$ were stage 3 , and $7(9 \%)$ were stage 4 . Esophageal varices, portal hypertension, and ascites were present in $18(23 \%)$ patients, while lower extremity edema was documented in $44(56 \%)$ patients. Hepatitis B surface antigen (HBsAg) and Hepatitis $\mathrm{C}$ virus antibody ( $\mathrm{HCV} \mathrm{Ab)}$ were checked in 60 and 65 patients, respectively, and were negative. All $79(100 \%)$ patients were on ursodeoxycholic acid. Few cases of psoriasis (5\%), CREST syndrome (5\%), Sjogren's syndrome $(8 \%)$, or rheumatoid arthritis $(9 \%)$ were noted. We did not find any associated cases of systemic lupus erythematosus or dermatomyositis.

The MESA source population grew modestly over the 20-year study timeframe, going from a low of 364,722 MESA person-years in 1992-1996 to a high of 409,670 person-years in 2007-2011 ( 0.6\% annual growth). The proportion of males and females in MESA was consistent throughout the 


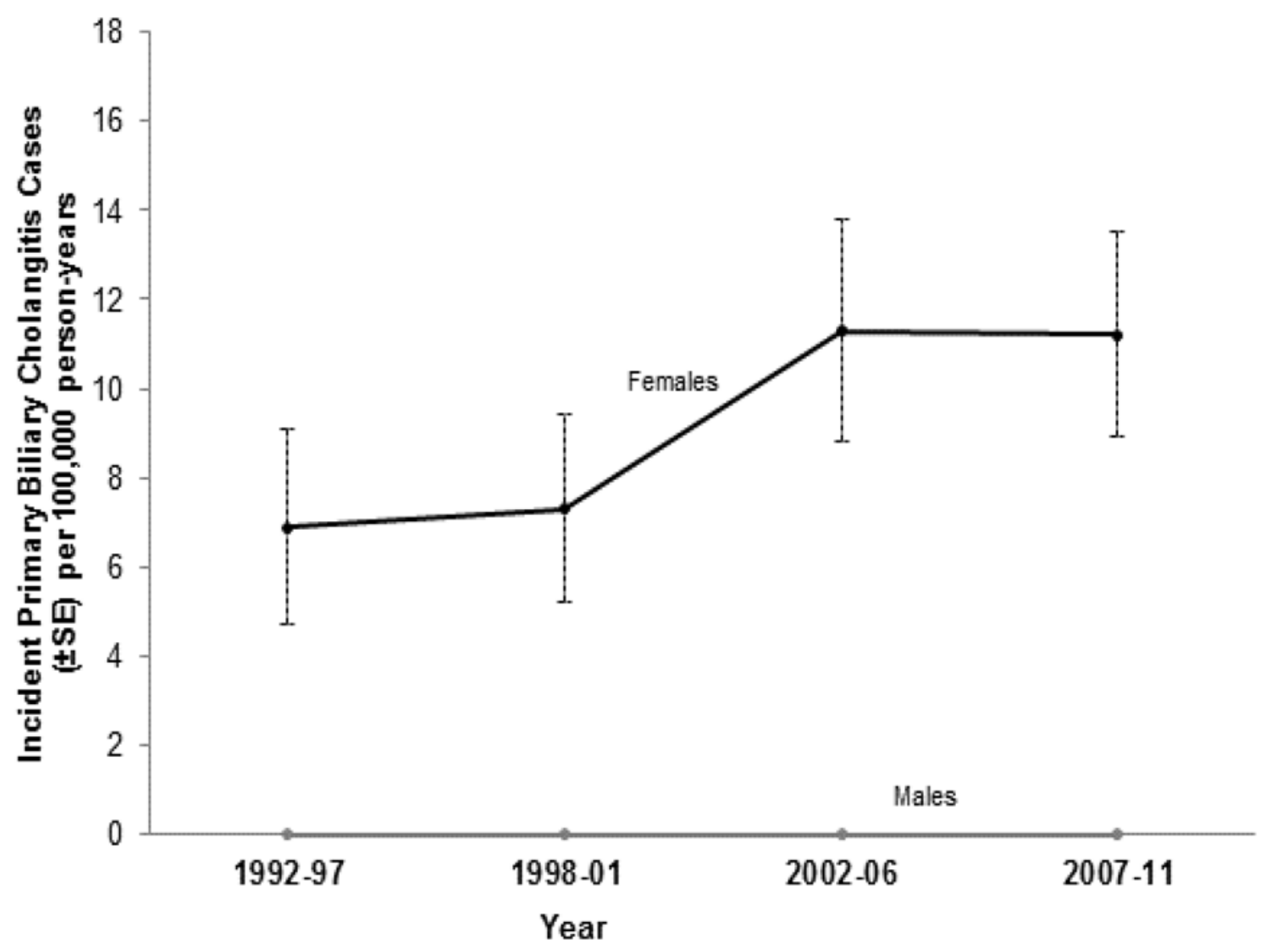

Figure 1. Age-standardized incidence rate of primary biliary cholangitis (PBC), stratified by gender, in 5-year time segments.

study timeframe, but there was a general population aging trend, with a $29 \%$ increase in the number of individuals age 40-69 years in 2007-2011 relative to 1992-1996.

The overall age- and sex-standardized PBC incidence rate was 4.9 cases per 100,000 person-years. The annual incidence rate of $\mathrm{PBC}$ increased, though not significantly $(P$ for trend $=$ 0.114 ), during the 20-year study timeframe. $\mathrm{PBC}$ incidence was sporadic for males. In females, $\mathrm{PBC}$ incidence ranged from a low of 6.9 [95\% confidence interval $(\mathrm{CI})=2.6-11.1$ ) cases per 100,000 person-years in $1992-1996$ to a high of 11.3 (95\% CI $=6.5-16.1)$ cases per 100,000 person-years in 2002-2006 (figure 1). Sex-specific comparisons were not significant between any 5-year time period.

After a mean $( \pm \mathrm{SD})$ of $7.3 \pm 5.2$ years of follow-up, $23(29 \%)$ patients with $\mathrm{PBC}$ were deceased. The survival curve for PBC patients identified in this study is shown in figure 2. The estimated 10-year survival of PBC cases in MESA was $76 \%$.

\section{Discussion}

Primary biliary cholangitis is characterized by T-lymphocyte mediated damage of the small interlobular bile ducts, resulting in cholestasis and the potential for cirrhosis and liver failure. Evidence suggests that the incidence and prevalence of $\mathrm{PBC}$ can vary considerably by time and geography. Only two studies have been done in the United States, with the most recent published over a decade ago. Here, we updated those findings in a Midwestern population captured by MESA.

Our findings are largely similar to those previously observed by Kim et al. ${ }^{12}$ The overall age- and sex-adjusted PBC incidence rate in this rural Midwestern population of Wisconsin was 4.9 cases per 100,000 person-years. This rate is slightly higher than the rate of 2.7 per 100,000 person-years found in Olmsted county Minnesota more than a decade ago. Though the annual incidence rate in females appeared to gradually increase over the course of two decades, increasing from 6.9 per 100,000 person-years in $1992-1996$ to 11.3 per 100,000 person-years in 2002-2006, this change was not statistically significant. As expected, PBC incidence was clearly higher in women than in men.

Although the incidence of $\mathrm{PBC}$ was higher, we observed better survival of individuals with PBC than in the 2000 report by Kim et al. ${ }^{12}$ The estimated 10-year survival of PBC cases in MESA was 76\%, compared to Olmsted county's PBC survival of $59 \% .^{11}$ Better survival could be due to various reasons, including better medical care in the more recent era and more routine use of ursodeoxycholic acid. Treatment 


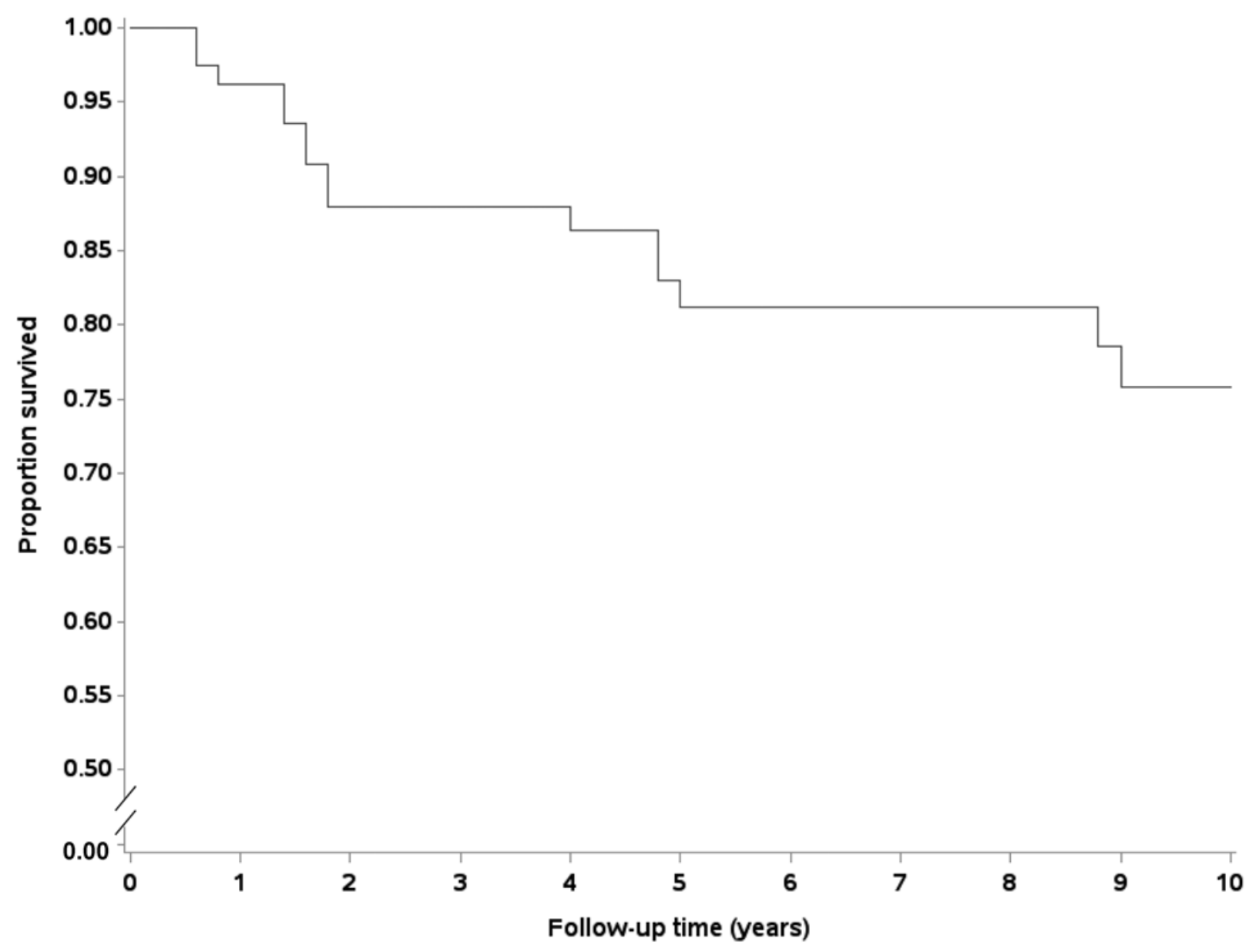

Figure 2. Kaplan-Meier survival estimates over 10-years for patient with primary biliary cholangitis (PBC).

with obeticholic acid was not included in this study, as it was not available in the market at the time of the study.

The present study is limited by the retrospective nature of the data collection, observational design, and inclusion of only a single center. Study limitations include those inherent to retrospective review of medical records, including the potential for incomplete or missing data and the relative rarity of the disease. Reliance on diagnoses recorded in the electronic medical record also makes it difficult to standardize diagnostic criteria, but manual review of all cases and exclusion of questionable diagnoses ensured standardization to the greatest extent possible in a retrospective study. Nevertheless, results suggest that the overall incidence of PBC in the United States is not rising quickly, and that patients with $\mathrm{PBC}$ have generally improved prognosis and survival.

In conclusion, the overall incidence of PBC in a Midwestern population of the United States has remained relatively stable over the last two decades. Patients have better prognosis, and the survival of PBC cases has improved.

\section{Acknowledgements}

The authors wish to thank the Marshfield Clinic Research Institute for study support, particularly Rachel V. Stankowski, $\mathrm{PhD}$ and Marie Fleisner in the Office of Scientific Writing and Publication for assistance with the manuscript.

\section{References}

1. Griffiths L, Dyson JK, Jones DE. The new epidemiology of primary biliary cirrhosis. Semin Liver Dis 2014;34:318-328.

2. Invernizzi P, Selmi C, Ranftler C, Podda M, Wesierska-Gadek J. Antinuclear antibodies in primary biliary cirrhosis. Semin Liver Dis 2005;25:298-310.

3. Triger DR, Berg PA, Rodes J. Epidemiology of primary biliary cirrhosis. Liver 1984;4:195 200.

4. Bhandari BM, Bayat H, Rothstein KD. Primary biliary cirrhosis. Gastroenterol Clin North Am 2011;40:373-386.

5. Delgado JS, Vodonos A, Delgado B, Jotkowitz A, Rosenthal A, Fich A, Novack V. Primary biliary cirrhosis in Southern Israel: a 20 year follow up study. Eur J Intern Med 2012;23:e193-e198.

6. Kumagi T, Onji M. Presentation and diagnosis of primary biliary cirrhosis in the 21st century. Clin Liver Dis 2008;12:243259. 
7. Beuers U, Gershwin ME, Gish RG, Invernizzi P, Jones DE, Lindor K, Ma X, Mackay IR, Parés A, Tanaka A, Vierling JM, Poupon R. Changing nomenclature for PBC: From 'cirrhosis' to 'cholangitis'. Hepatology 2015;62:1620-1622.

8. Lindor KD, Gershwin ME, Poupon R, Kaplan M, Bergasa NV, Heathcote EJ; American Association for Study of Liver Diseases. Primary biliary cirrhosis. Hepatology 2009;50:291 308.

9. Selmi C, Mayo MJ, Bach N, Ishibashi H, Invernizzi P, Gish RG, Gordon SC, Wright HI, Zweiban B, Podda M, Gershwin ME. Primary biliary cirrhosis in monozygotic and dizygotic twins: genetics, epigenetics, and environment. Gastroenterology 2004;127:485-492.

10. Danielsson A, Boqvist L, Uddenfeldt P. Epidemiology of primary biliary cirrhosis in a defined rural population in the northern part of Sweden. Hepatology 1990;11:458-464.

11. James OF, Bhopal R, Howel D, Gray J, Burt AD, Metcalf JV. Primary biliary cirrhosis once rare, now common in the United Kingdom? Hepatology 1999;30:390-394.

12. Kim WR, Lindor KD, Locke GR 3rd, Therneau TM, Homburger HA, Batts KP, Yawn BP, Petz JL, Melton LJ 3rd, Dickson ER. Epidemiology and natural history of primary biliary cirrhosis in a US community. Gastroenterology 2000;119:1631-1636.

13. Kingham JG, Parker DR. The association between primary biliary cirrhosis and coeliac disease: a study of relative prevalences. Gut 1998;42:120-122.

14. Löfgren J, Järnerot G, Danielsson D, Hemdal I. Incidence and prevalence of primary biliary cirrhosis in a defined population in Sweden. Scand J Gastroenterol 1985;20:647650.

15. Metcalf JV, Bhopal RS, Gray J, Howel D, James OF. Incidence and prevalence of primary biliary cirrhosis in the city of Newcastle upon Tyne, England. Int J Epidemiol 1997;26:830 836.

16. Rautiainen $H$, Salomaa V, Niemelå $S$, Karvonen AL, Nurmi H, Isoniemi H, Farkkila M. Prevalence and incidence of primary biliary cirrhosis are increasing in Finland. Scand $\mathbf{J}$ Gastroenterol 2007;42:1347-1353.

17. Hurlburt KJ, McMahon BJ, Deubner H, Hsu-Trawinski B, Williams JL, Lowdley KV. Prevalence of autoimmune liver disease in Alaska Natives. Am J Gastroenterol 2002;97:24022407.

18. Kieke AL, Kieke BA Jr, Kopitzke SL, McClure DL, Belongia EA, VanWormer JJ, Greenlee RT. Validation of Health Event Capture in the Marshfield Epidemiologic Study Area. Clin Med Res 2015;13:103-111.

19. DeStefano F, Eaker ED, Broste SK, Peissig PL, Vierkant RA, Konitzer KA, Gruber RL, Layde PM. Epidemiologic research in an integrated regional medical care system: the Marshfield Epidemiologic Study Area. J Clin Epidemiol 1996;49:643-652.

\section{Author Affiliations}

Rajan Kanth, MD*; Ram Babu Shrestha, MS广; Indira Rai, MDf; Jeffrey J. VanWormer, PhD\$; Praveen K. Roy, MD\#

*At the time the study was conducted, Dr. Kanth was a hospitalist at the Marshfield Clinic, Marshfield, Wisconsin USA. Current affiliation: Carilion Clinic, Department of Gastroenterology, Roanoke, Virginia USA

HIntegrated Research and Development Laboratory, Marshfield Clinic Research Institute, Marshfield, Wisconsin USA

‡Department of Internal Medicine, Marshfield Clinic, Marshfield, Wisconsin USA

$\S$ Center for Clinical Epidemiology \& Population Health, Marshfield Clinic Research Institute, Marshfield, Wisconsin USA

\#Department of Gastroenterology, Presbyterian Healthcare Services, Albuquerque, New Mexico USA 